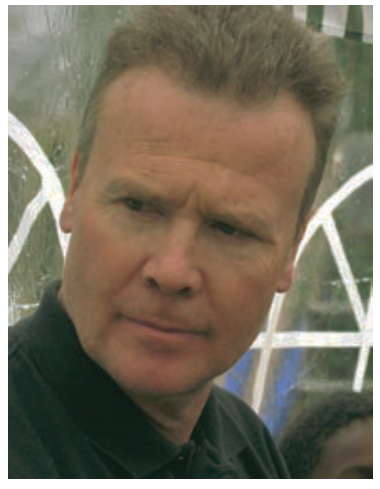

G. Dine

\section{Progression analytique et diversité des applications}

\begin{abstract}
La biologie clinique, aujourd'hui, permet d'assurer $60 \%$ à $70 \%$ des diagnostics médicaux selon les dernières études objectives réalisées. Cela sous-entend, au-delà de l'affirmation d'une pathologie chez un patient, que la prise en charge médicale et donc les traitements nécessaires vont découler des résultats biologiques. C'est une responsabilité énorme qui nécessite la fiabilité et la reproductibilité des analyses dans un contexte de qualité incontournable. De grands efforts ont été accomplis depuis 15 ans. La réglementation a encadré la démarche professionnelle avec des procédures consensuelles mais également référentes qui s'imposent à tous les biologistes quel que soit leur exercice. Dans ce contexte, les mises au point régulières fournies par la HAS sont autant de repères utiles dans un environnement évolutif rapide, notamment au plan technologique. En effet, si la biologie clinique est devenue aussi performante, c'est parce que les outils qu'elle met à disposition se rapprochent de la réalité physiologique du fonctionnement de notre corps et permettent l'identification des mécanismes pathologiques du point de vue de la cellule mais de plus en plus du point de vue de la molécule ou du gène, eux-mêmes. Le concept de thérapies ciblées a déjà été abordé dans les numéros précédents de Biotribune Magazine. La réflexion - une maladie, un diagnostic, un traitement - est en passe de devenir une routine de travail, en dehors, bien sûr, des polypathologies, notamment des personnes âgées. Pour utiliser un langage d'ingénieur, l'asservissement entre la proposition thérapeutique et la réalité du dysfonctionnement identifié par l'introspection biologique va devenir habituel pour les différentes professions de santé au sein d'une chaîne de prise en charge des patients. Certains systèmes complexes de prises d'informations biologiques deviennent utilisables au coup par coup et à proximité du patient pour affirmer des diagnostics difficiles à réaliser parfois en urgence et pour permettre l'utilisation de traitements à grande échelle dont l'impact sur la morbidité et la mortalité n'est plus discutable. II n'en reste pas moins qu'un écueil, aujourd'hui, dans nos sociétés occidentales, doit rester à l'esprit, c'est le coût induit afin de permettre à nos systèmes de prestations de suivre l'escalade du progrès et ses bénéfices et de ne pas le réserver à une catégorie de populations favorisées. Dans ces conditions, l'interaction entre sciences de la vie, sciences de la santé et sciences de l'ingénieur est non seulement une réalité mais va s'amplifier. La collaboration des compétences apparaît de plus en plus évidente à la lecture de plusieurs articles de notre numéro 32. Pour les biologistes, la compréhension des progrès scientifiques mais surtout technologiques doit leur permettre de faire les meilleurs choix du point de vue de leurs acquisitions eu égard à leurs pratiques professionnelles au sein des laboratoires qu'ils dirigent. Toutefois, quels que soient ces progrès qui peuvent donner le vertige en termes d'accélération, il n'en demeure pas moins que nos métiers s'exercent à destination de patients, c'està-dire d'individus. Notre démarche ne doit pas l'ignorer et intégrer, au-delà des réflexions objectives sur la technicité, la qualité et le coût, la dimension humaine et ses différentes composantes, notamment psychologiques. Pour ceux qui craignent la technologie toute puissante, il faut bien penser que dans ces conditions, les ingénieurs ne remplaceront pas les professions de santé qui resteront à l'interface avec les patients. Par contre, du point de vue de la biologie clinique, nous devons maîtriser toutes les problématiques exposées précédemment mais il apparaît de plus en plus évident que le métier s'orientera vers une nécessité de service, au risque d'être court-circuité. Les exemples issus des progrès constatés dans l'exploration plaquettaire le démontrent. La mise à disponibilité d'outils accessibles à la pratique quotidienne de cliniciens rompus à l'exercice critique et urgent doit permettre aux biologistes de conquérir de nouveaux terrains d'expression professionnelle. •
\end{abstract}

G. Dine 